\title{
A Method to Selectively Observe a Desired Linear Combination of Chemical Shifts in GFT Projection NMR Spectroscopy
}

\author{
Monalisa Swain ${ }^{\mathrm{a}, \mathrm{b}}$ and Hanudatta S. Atreya*,b \\ ${ }^{a}$ Solid State and Structural Chemistry Unit and ${ }^{b}$ NMR Research Centre, Indian Institute of Science, Bangalore-560012, \\ India
}

\begin{abstract}
In recent years, several new methods have emerged for rapid NMR data collection. One class of methods is projection NMR spectroscopy, which involves phase sensitive joint sampling of two or more chemical shifts in an indirect dimension of a multidimensional NMR experiment. In the current implementations of this method, cosine/sine modulation of all chemical shifts involved in the joint sampling are collected and stored as separate FIDs. A post-acquisition data processing step (application of G-matrix) then separates the different inter-modulations of chemical shifts. Thus, joint sampling of $K+1$ spins results in $2^{K}$ combination of chemical shifts (also representing $2^{K}$ projection angles). One limitation of this approach is that even if only a few of the $2^{K}$ components of the multiplet (or projection angles) is desired, an entire data set containing information for all $2^{K}$ shift combinations is collected. We propose here a simple method which releases this restriction and allows one to selectively detect only the desired linear combination of chemical shifts/projection angles out of $2^{K}$ combinations in a phase sensitive manner. The method involves selecting the appropriate cosine/sine modulations of chemical shifts and forming the desired linear combination by phase cycling of the radiofrequency pulses and receiver. This will benefit applications where only certain linear combination of shifts are desired or/and are sufficient. Further, G-matrix transformation required for forming the linear combination is performed within the pulse sequence. This avoids the need for any post-acquisition data processing. Taken together, this mode of data acquisition will foster new applications in projection NMR spectroscopy for rapid resonance assignment and structure determination.
\end{abstract}

Keywords: Projection NMR spectroscopy, GFT NMR, fast NMR methods, phase cycling, resonance assignments.

\section{INTRODUCTION}

Structure determination of proteins and other biomolecules by NMR spectroscopy has undergone a revolution with the advent of several new methods overcoming the problems of sensitivity and resolution [1]. Supported by these advancements, a new dimension in NMR research has emerged which aims to increase the speed with which data is collected and analyzed [2]. This is useful for systems in the 'sampling-limited' regime [2], wherein the measurement time required for data collection becomes the rate-limiting step for structural characterization. One class of methods for rapid data collection is projection NMR spectroscopy in which the information of a high-dimensional spectrum is encoded in lower spectral dimensions [2,3]. The reduction in dimensionality is achieved by joint sampling of two or more chemical shifts in a single indirect dimension of a multidimensional NMR experiment. This results, after data processing, in different sub-spectra, each containing peaks with a specific linear combination of the jointly detected chemical shifts $[2,3]$. The different methods based on this principle are reduced dimensionality NMR (RD-NMR) [4], G-matrix Fourier transform NMR (GFT-NMR) [5, 6] and Projection-reconstruction NMR (PR-NMR) [7]. In the PRNMR method, the different linear combinations of chemical shifts are denoted by specific projection angles [3, 7]. The projected subspectra (recorded with different scaling of the

*Address correspondence to this author at the NMR Research Centre, Indian Institute of Science, Bangalore-560012, India; Tel: +91-80-2293-3302; Fax: +91-80-2360-1550; E-mail: hsatreya@ sif.iisc.ernet.in chemical shift evolution periods) are then used to 'reconstruct' the parent $N \mathrm{D}$ spectrum [7]. Techniques to automate the process of acquiring, processing and analyzing projection NMR data have also been proposed [8, 9]. All of these methods have been now been demonstrated for rapid resonance assignment of globular [10], membrane [11] and unfolded proteins [12].

In all the projection NMR methods, joint sampling in the indirect dimension is achieved by co-incrementing two or more chemical shift evolution periods in the radio frequency (r.f.) pulse sequence, with the increments being scaled relative to each other [2-7]. This results in signals comprising linear combination of the respective chemical shifts that are jointly sampled. In the case of RD-NMR, only one of the shifts in this set is sampled in a phase sensitive manner [4]. That is, real and imaginary components of the free induction decay (FID) are acquired only for one of the nuclei in the jointly sampled set of chemical shifts. In the more recent GFT-NMR and PR-NMR methods, quadrature components of all the jointly sampled shifts are collected [5-7]. Thus, if two nuclei $\mathrm{A}$ and B with chemical shifts $\Omega_{\mathrm{A}}$ and $\Omega_{\mathrm{B}}$ are jointly sampled with relative scaling factors $\kappa_{A}$ and $\kappa_{B}$ (where $\kappa$ is a scaling factor [5] equivalent to a 'projection angle' in PR-NMR [3, 7]), four different FIDs are collected separately with each FID having the following transfer amplitudes $[2,5]$ :

$\begin{aligned} & \mathrm{S}_{1 \mathrm{r}}=\cos \left(\kappa_{\mathrm{A}} * \Omega_{\mathrm{A}} * \mathrm{t}\right) * \cos \left(\kappa_{\mathrm{B}} * \Omega_{\mathrm{B}} * \mathrm{t}\right) \\ & \mathrm{S}_{1 \mathrm{i}}=\sin \left(\kappa_{\mathrm{A}} * \Omega_{\mathrm{A}} * \mathrm{t}\right) * \cos \left(\kappa_{\mathrm{B}} * \Omega_{\mathrm{B}} * \mathrm{t}\right) \\ & \mathrm{S}_{2 \mathrm{r}}=\cos \left(\kappa_{\mathrm{A}} * \Omega_{\mathrm{A}} * \mathrm{t}\right) * \sin \left(\kappa_{\mathrm{B}} * \Omega_{\mathrm{B}} * \mathrm{t}\right) \\ & \mathrm{S}_{2 \mathrm{i}}=\sin \left(\kappa_{\mathrm{A}} * \Omega_{\mathrm{A}} * \mathrm{t}\right) * \sin \left(\kappa_{\mathrm{B}} * \Omega_{\mathrm{B}} * \mathrm{t}\right)\end{aligned} \quad \Rightarrow \mathbf{S}(2)=\left[\begin{array}{c}S_{1 r} \\ S_{1 i} \\ S_{2 r} \\ S_{2 i}\end{array}\right]$ 
These FIDs are stored separately and the application of G-matrix transformation [5] in a post-acquisition manner allows the editing of the linear combination of chemical shifts as follows:

$$
\begin{aligned}
& \mathbf{G}(2)=\left[\begin{array}{cccc}
1 & 0 & 0 & -1 \\
0 & 1 & 1 & 0 \\
1 & 0 & 0 & 1 \\
0 & 1 & -1 & 0
\end{array}\right] \\
& \mathbf{T}(2)=\mathbf{G}(2) \cdot \mathbf{S}(2)=\left[\begin{array}{c}
\cos \left(\kappa_{A} \Omega_{A}+\kappa_{B} \Omega_{B}\right) t \\
\sin \left(\kappa_{A} \Omega_{A}+\kappa_{B} \Omega_{B}\right) t \\
\cos \left(\kappa_{A} \Omega_{A}-\kappa_{B} \Omega_{B}\right) t \\
\sin \left(\kappa_{A} \Omega_{A}-\kappa_{B} \Omega_{B}\right) t
\end{array}\right]= \\
& {\left[\begin{array}{c}
e^{i\left(\kappa_{A} \Omega_{A}+\kappa_{B} \Omega_{B}\right) t} \\
e^{i\left(\kappa_{A} \Omega_{A}-\kappa_{B} \Omega_{B}\right) t}
\end{array}\right]}
\end{aligned}
$$

As a result, the 2 linear combinations $\kappa_{\mathrm{A}} * \Omega_{\mathrm{A}} * \pm \kappa_{\mathrm{B}} * \Omega_{\mathrm{B}}$ (which also represent projections at angles $\pm \alpha=\tan ^{-1}\left(\kappa_{\mathrm{B}} / \kappa_{\mathrm{A}}\right)$ $[3,7])$ are measured in one of each of the 2 subspectra. This methodology can be extended for joint sampling of more than two spins. Thus, in a generalized case of a $(N, N-K) \mathrm{D}$ projection NMR experiment, if one chooses a particular chemical shift denoted $\Omega_{0}$ out of the set of $K+1$ jointly sampled shifts as the phase-sensitively detected 'center shift', sampling of the remaining $K$ shifts, $\Omega_{1}, \Omega_{2} \ldots . . \Omega_{K}$, generates chemical shift multiplets centered about $\Omega_{0}$ and the linear combinations $\kappa_{0} \Omega_{0} \pm \kappa_{2} \Omega_{1} \ldots \pm \kappa_{K} \Omega_{K}$ (projections at angles: $\left.\pm \alpha_{1}, \pm \alpha_{2} \ldots \pm \alpha_{K}\right)$ are measured. While jointly incrementing $K+1$ shift evolution periods, the phases $\Phi_{j}$ of the r.f. pulses exciting spins of type $j(j=1 \ldots K)$ are systematically varied between $0^{\circ}$ and $90^{\circ}$ in order to register both cosine and sine modulated data sets $[2,3,5,6]$. This results in $2^{K+1}$ FIDs which upon G-matrix transformation yields $2^{K}$ 'basic spectra', and each of these sub-spectra affords editing of the shift components.

One limitation of the above method is that during each data collection, quadrature components of all the jointly sampled shifts have to be collected and stored separately. This results in an increased measurement time when only one particular or a few of the linear combination/projection out of the $2^{K}$ linear combinations is desired. In other words, even when only one of the $2^{K}$ components of the multiplet is needed, an entire data set containing information for all $2^{K}$ shift combinations is collected. For instance, in a given (4, 2)D GFT experiment, only a particular linear combination, say $\kappa_{1} * \Omega_{1}+\kappa_{2} * \Omega_{2}-\kappa_{3} * \Omega_{3}$ (projection at an angle $\left(+\alpha_{1},-\beta_{1}\right) ; \alpha$ $=\tan ^{-1}\left(\kappa_{2} / \kappa_{1}\right)$ and $\beta_{1}=\tan ^{-1}\left(\kappa_{3} / \sqrt{ }\left(\kappa_{1}^{2}+\kappa_{2}^{2}\right)\right.$ [3]), may be of interest and other linear combinations may not be required. Alternatively, two different linear combination such as $\kappa_{1} * \Omega_{1}+\kappa_{2} * \Omega_{2}+\kappa_{3} * \Omega_{3}$ and $\kappa_{1}{ }^{\prime} * \Omega_{1}+\kappa_{2}{ }^{*} * \Omega_{2}+\kappa_{3}{ }^{*} * \Omega_{3} \quad\left(\kappa_{1}, \neq \kappa_{1}\right.$; $\kappa_{2}, \neq \kappa_{2} ; \kappa_{3}, \neq \kappa_{3}$ ) may be desired. In the current implementations, such selective detection is not possible and data has to be collected and stored for all the four (or eight) linear combinations: $\kappa_{1} * \Omega_{1} \pm \kappa_{2} * \Omega_{2} \pm \kappa_{3} * \Omega_{3}$ (i.e., projections at angles $\left.\left( \pm \alpha_{1}, \pm \beta_{1}\right)\right)$ and $\kappa_{1}{ }^{\prime} * \Omega_{1} \pm \kappa_{2}{ }^{\prime} * \Omega_{2} \pm \kappa_{3}, * \Omega_{3}$. We propose here a simple method based on phase cycling which releases this restriction and allows one to acquire projection NMR data comprising any given specific linear combination out of $2^{K}$ shift combinations. Instead of collecting and storing the FIDs separately, appropriate cosine/sine modulations of chemical shifts are selected and the desired linear combination is constructed by phase cycling of the radiofrequency pulses and receiver. In all the experiments, the existing 2 or more phase cycling steps in the r.f. sequence for water/artifact suppression can be utilized to incorporate this phase cycling procedure. This method implements G-matrix transformation within the r.f. pulse sequence and hence avoids the need for any post-acquisition data processing. The spectra encoding sums and differences of chemical shifts can be directly visualized by the spectroscopist without the need of extra processing steps. The method can be applied to any projection NMR experiment employing the States method [13] or the sensitivity-enhanced/echo-antiecho method [14] for quadrature detection. We demonstrate this method for three experiments: (i) $(3,2) \mathrm{D}$ HACA(CON)HN [5], (ii) $(3,2) \mathrm{D}$ HNNCO [2] and (iii) $(4,2) \mathrm{D}$ HNNCOCA. For the nuclei shown underlined, chemical shifts are jointly sampled $[2,4$, 5]. The first experiment employs the States method [13] for quadrature detection, whereas the other two serve to demonstrate the method on experiments employing the sensitivityenhanced scheme of data acquisition. Applications where this method will be useful are also discussed.

\section{MATERIALS AND METHODOLOGY}

\section{Theory}

The method can be understood by considering a set of two jointly sampled spins, $A$ and $B$ having chemical shifts $\Omega_{\mathrm{A}}$ and $\Omega_{\mathrm{B}}$ in a $(N, N-1) \mathrm{D}$ NMR experiment (e.g., a $(3,2) \mathrm{D}$ or a $(4,3) \mathrm{D}$ experiment). Consider that a phase of ' $x$ ' for the r.f pulse exciting spins $A\left(\Phi_{A}\right)$ or $B\left(\Phi_{B}\right)$ results in cosine modulation of their chemical shifts and a phase of ' $y$ ' results in their corresponding sine modulations. Consider also that an $180^{\circ}$ phase shifts of these r.f. pulses $(x \rightarrow-x ; y \rightarrow-y)$ results in an inversion of the signals. Any one out of the two possible linear combinations, $\kappa_{\mathrm{A}} * \Omega_{\mathrm{A}} * \pm \kappa_{\mathrm{B}} * \Omega_{\mathrm{B}}$, can be selectively chosen to be observed using a phase cycling scheme involving with 2 scans or transients for each increment. In the PR-NMR nomenclature, this implies that out of the two projection angles, $\pm \alpha$, for a $(3,2) \mathrm{D}$ experiment chemical shifts at either $+\alpha$ or $-\alpha$ projections can be selectively chosen for observation. The phase cycling steps are shown in Tables 1 and 2. Thus, for linear combination, $\kappa_{\mathrm{A}} * \Omega_{\mathrm{A}}+\kappa_{\mathrm{B}} * \Omega_{\mathrm{B}}$ (Table 1), FID $1=\cos \left(\left(\kappa_{\mathrm{A}} * \Omega_{\mathrm{A}}+\kappa_{\mathrm{B}} * \Omega_{\mathrm{B}}\right) t\right)$ and FID2= $\sin \left(\left(\kappa_{\mathrm{A}} * \Omega_{\mathrm{A}}+\kappa_{\mathrm{B}} * \Omega_{\mathrm{B}}\right) t\right)$ for each increment can be collected and treated as the real and imaginary components like in any generic 2D NMR experiment. The Fourier transformation of the complex signal will then result in the direct observation of only the linear combination: $\kappa_{\mathrm{A}} * \Omega_{\mathrm{A}}+\kappa_{\mathrm{B}} * \Omega_{\mathrm{B}}$ in the indirect dimension. In the case of the other linear combination $\left(\kappa_{\mathrm{A}} * \Omega_{\mathrm{A}}-\kappa_{\mathrm{B}} * \Omega_{\mathrm{B}}\right.$; Table 2), FID $1=\cos \left(\left(\kappa_{\mathrm{A}} * \Omega_{\mathrm{A}}-\kappa_{\mathrm{B}} * \Omega_{\mathrm{B}}\right) t\right)$ and FID2 $=\sin \left(\left(\kappa_{\mathrm{A}} * \Omega_{\mathrm{A}}-\kappa_{\mathrm{B}} * \Omega_{\mathrm{B}}\right) t\right)$ for each increment can be collected. The Fourier transformation of the complex signal will result in the direct observation of only the linear combination: $\kappa_{\mathrm{A}} * \Omega_{\mathrm{A}}-\kappa_{\mathrm{B}} * \Omega_{\mathrm{B}}$ in the indirect dimension. In case if two or more scans are already used in acquiring the data for effective water/artifact suppression [1], they can be utilized in the present method thereby avoiding the need to increase the number of steps in the phase cycle. 
Table 1. Selective Observation of $\kappa_{A} * \Omega_{A}+\kappa_{B} * \Omega_{B}$

\begin{tabular}{|c|c|c|c|}
\hline & \multicolumn{2}{|c|}{ Phases of r.f. Pulses } & $\begin{array}{c}\text { Receiver } \\
\text { Phase Cycle }^{\mathrm{a}}\end{array}$ \\
\hline & Scan 1 & Scan 2 & Scan 1 Scan 2 \\
\hline $\begin{array}{l}\text { FID } 1 \\
\text { (real) }\end{array}$ & $\begin{array}{c}\Phi_{A}=x \Phi_{B}=x \\
\cos \left(\kappa_{A} \Omega_{\mathrm{A}} \mathrm{t}\right) * \cos \left(\kappa_{\mathrm{B}} \Omega_{\mathrm{B}} \mathrm{t}\right)\end{array}$ & $\begin{array}{c}\Phi_{A}=-y \Phi_{B}=y \\
-\sin \left(\kappa_{\mathrm{A}} \Omega_{\mathrm{A}} \mathrm{t}\right) * \sin \left(\kappa_{\mathrm{B}} \Omega_{\mathrm{B}} \mathrm{t}\right)\end{array}$ & $\begin{array}{c}x \quad x \\
=\cos \left(\kappa_{\mathrm{A}} \Omega_{\mathrm{A}}+\kappa_{\mathrm{B}} \Omega_{\mathrm{B}}\right) t\end{array}$ \\
\hline $\begin{array}{c}\text { FID } 2 \\
(\mathrm{imag}) \\
\left(\Phi_{A}=\Phi_{A}+90^{\circ}\right)\end{array}$ & $\begin{array}{c}\Phi_{A}=y \Phi_{B}=x \\
\sin \left(\kappa_{\mathrm{A}} \Omega_{\mathrm{A}} \mathrm{t}\right) * \cos \left(\kappa_{\mathrm{B}} \Omega_{\mathrm{B}} \mathrm{t}\right)\end{array}$ & $\begin{array}{c}\Phi_{A}=x \Phi_{B}=y \\
\cos \left(\kappa_{\mathrm{A}} \Omega_{\mathrm{A}} \mathrm{t}\right) * \sin \left(\kappa_{\mathrm{B}} \Omega_{\mathrm{B}} \mathrm{t}\right)\end{array}$ & $\begin{array}{c}x \quad x \\
=\sin \left(\kappa_{\mathrm{A}} \Omega_{\mathrm{A}}+\kappa_{\mathrm{B}} \Omega_{\mathrm{B}}\right) t\end{array}$ \\
\hline
\end{tabular}

${ }^{a}$ The receiver phase cycle can be adjusted accordingly to either add or subtract signals from the two scans. That will depend on the exact implementation of the experiment and requirement for water/artifact suppression. Thus, a two step receiver phase cycle of $(x,-x)$ will lead to subtraction of the signals from the two scans. The r.f. phases then have to be adjusted accordingly to form the correct linear combination. For instance, if receiver phase cycle of $(x,-x)$ is needed, Scan $1: \Phi_{A}=x$ and $\Phi_{B}=x$ and Scan 2 : $\Phi_{A}=y$ and $\Phi_{B}=y$ could be used. Note that in existing implementations, four FIDs each with 2 scans ( 8 scans) are needed to form the linear combination $\kappa_{\mathrm{A}} * \Omega_{\mathrm{A}}+\kappa_{\mathrm{B}}{ }^{*} \Omega_{\mathrm{B}}($ Eq. $(1)$; see Results and Discussion for comparison of the measurement times).

Table 2. Selective Observation of $\kappa_{A} * \Omega_{A}-\kappa_{B} * \Omega_{B}$

\begin{tabular}{|c|c|c|c|}
\hline & \multicolumn{2}{|c|}{ Phases of r.f. Pulses } & $\begin{array}{c}\text { Receiver } \\
\text { Phase Cycle }\end{array}$ \\
\hline & Scan 1 & Scan 2 & Scan 1 Scan 2 \\
\hline $\begin{array}{l}\text { FID } 1 \\
\text { (real) }\end{array}$ & $\begin{array}{c}\Phi_{A}=x \Phi_{B}=x \\
\cos \left(\kappa_{\mathrm{A}} \Omega_{\mathrm{A}} \mathrm{t}\right) * \cos \left(\kappa_{\mathrm{B}} \Omega_{\mathrm{B}} \mathrm{t}\right)\end{array}$ & $\begin{array}{c}\Phi_{A}=y \Phi_{B}=y \\
\sin \left(\kappa_{\mathrm{A}} \Omega_{\mathrm{A}} \mathrm{t}\right)^{*} \sin \left(\kappa_{\mathrm{B}} \Omega_{\mathrm{B}} \mathrm{t}\right)\end{array}$ & $\begin{array}{cc}x & x \\
=\cos \left(\kappa_{\mathrm{A}} \Omega_{\mathrm{A}}-\kappa_{\mathrm{B}} \Omega_{\mathrm{B}}\right) t\end{array}$ \\
\hline
\end{tabular}

The methodology described above can be extended to any general $(N, N-K) \mathrm{D}$ projection NMR experiment. The procedure involves the following steps:

(i) Choose the r.f pulses exciting the spins of interest.

(ii) Estimate the change in the sign of the signals when the phase of these r.f. pulses is varied by $90^{\circ}$.

(iii) Determine the different cosine and sine modulations of the chemical shifts that are required to form a given linear combination of chemical shifts (or projection angle). For example, in joint sampling of two spins, $A$ and $B$ discussed above, to directly observe a linear combination such as $\kappa_{\mathrm{A}} * \Omega_{\mathrm{A}}+\kappa_{\mathrm{B}} * \Omega_{\mathrm{B}}$, $\cos \left(\kappa_{\mathrm{A}} \Omega_{\mathrm{A}} \mathrm{t}\right) \cos \left(\kappa_{\mathrm{B}} \Omega_{\mathrm{B}} \mathrm{t}\right)$ and $-\sin \left(\kappa_{\mathrm{A}} \Omega_{\mathrm{A}} \mathrm{t}\right) \sin \left(\kappa_{\mathrm{B}} \Omega_{\mathrm{B}} \mathrm{t}\right)$ need to be combined for the real part (i.e., $\left.\cos \left(\kappa_{\mathrm{A}} * \Omega_{\mathrm{A}}+\kappa_{\mathrm{B}} * \Omega_{\mathrm{B}}\right)\right)$. For the imaginary part, $\sin \left(\kappa_{\mathrm{A}} * \Omega_{\mathrm{A}}+\kappa_{\mathrm{B}} * \Omega_{\mathrm{B}}\right): \quad \sin \left(\kappa_{\mathrm{A}} \omega_{\mathrm{A}} \mathrm{t}\right) \cos \left(\kappa_{\mathrm{B}} \Omega_{\mathrm{B}} \mathrm{t}\right) \quad$ and $\cos \left(\kappa_{A} \Omega_{A} t\right) \sin \left(\kappa_{B} \Omega_{B} t\right)$ need to be combined. These modulations can be combined in the 2 scans/ transients of the phase cycle as shown in Table $\mathbf{1}$. The receiver phase cycle can be set to either add or subtract the signals from the two scans. (iv) One of the spins in the jointly sampled set of spins is chosen such that the r.f. phase of its excitation pulse is shifted by $90^{\circ}$ in all the scans to collect an additional FID which is treated as the imaginary part (e.g., FID 2 in Tables $\mathbf{1}$ and 2). This is similar to the procedure used in a standard 2D NMR experiment wherein complex signals are acquired [1].

(v) A standard Fourier transform of the resulting complex data (FID1 $+i$ FID2) is performed to directly observe the desired linear combination.

This method is also applicable to experiments involving sensitivity-enhanced scheme of data collection [1]. In these experiments, the chemical shift modulation of ${ }^{15} \mathrm{~N}$ signal can be controlled in two ways by using: (i) the r.f. pulse bringing the ${ }^{15} \mathrm{~N}$ longitudinal magnetization into transverse plane immediately before commencing its chemical shift evolution. An ' $x$ ' or ' $y$ ' phase for this pulse results in selection of cosine or sine modulation of the chemical shift and (ii) the r.f. pulse which is inverted in concert with a coherence selection gradient in order to transfer both the cosine and sine modulations of ${ }^{15} \mathrm{~N}$ chemical shifts to observable magnetization 


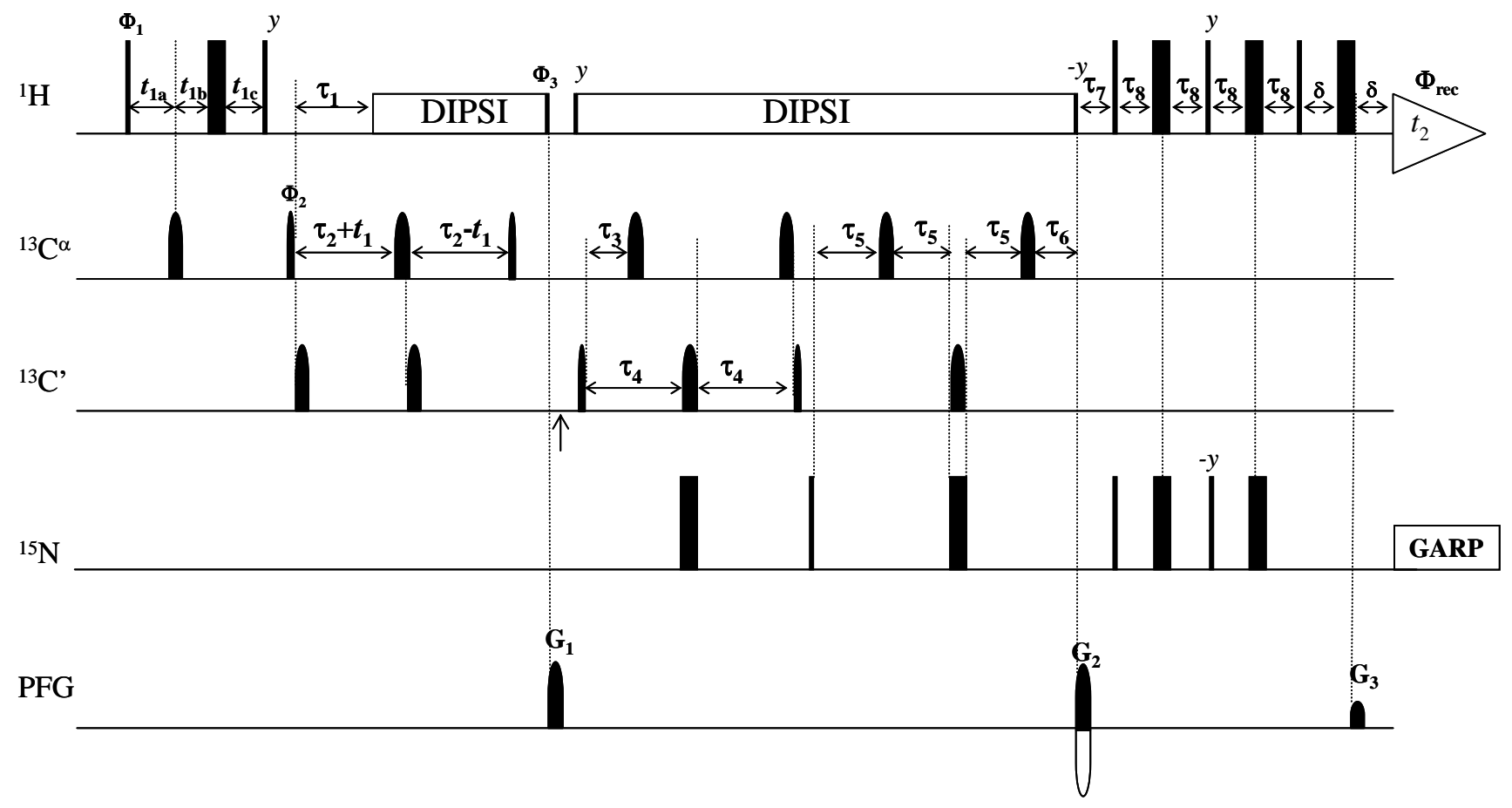

Fig. (1). R.f. pulse scheme of GFT $(3,2) \mathrm{D}$ HACA$(\mathrm{CON}) \mathrm{HN}$ employing the States method [13] for quadrature detection. Rectangular $90^{\circ}$ and $180^{\circ}$ pulses are indicated by thin and thick vertical bars, respectively, and phases are indicated above the pulses. Where no r.f. phase is marked, the pulse is applied along $x$. High-power $90^{\circ}$ pulse lengths are: $8.0 \mu \mathrm{s}$ for ${ }^{1} \mathrm{H}, 14.5 \mu \mathrm{s}$ for ${ }^{13} \mathrm{C}$ and $38 \mu \mathrm{s}$ for ${ }^{15} \mathrm{~N}$. The scaling factor: $\kappa=1.0$. The ${ }^{1} \mathrm{H}$ r.f. carrier is placed at the position of the solvent line at $4.7 \mathrm{ppm}$. The ${ }^{15} \mathrm{~N}$ carrier position is set to $119.5 \mathrm{ppm}$. The ${ }^{13} \mathrm{C}$ carrier is initially placed at $54 \mathrm{ppm}$ and switched to $176 \mathrm{ppm}$ before the first $90^{0} \mathrm{rf}$ pulse on ${ }^{13} \mathrm{CO}$ (indicated by an arrow). All ${ }^{13} \mathrm{C}$ pulses are of Guassian cascade shape having a pulse width of $320 \mu \mathrm{s}$ for $90^{\circ}$ (on- or off- resonance) and $220 \mu \mathrm{s}$ for $180^{\circ}$ (on- or off-resonance). DIPSI [1] (r.f $=3.2 \mathrm{kHz}$ ) is used for ${ }^{1} \mathrm{H}$ decoupling during ${ }^{13} \mathrm{C}-{ }^{13} \mathrm{C}$ and ${ }^{13} \mathrm{C}-{ }^{15} \mathrm{~N}$ polarization transfer. GARP [1] is employed to decouple ${ }^{15} \mathrm{~N}$ (r.f. $=1.50$ $\mathrm{kHz}$ ) during acquisition. The chemical shift evolution of ${ }^{1} \mathrm{H}$ is carried out in a semi-constant time manner [1] with the initial delay periods: $t_{1 \mathrm{a}}=1.8 \mathrm{~ms}, t_{1 \mathrm{~b}}=3 \mu \mathrm{s}, t_{1 \mathrm{c}}=1.8 \mathrm{~ms}$ and incremental delay: $\Delta t_{1 \mathrm{a}}=1 / 2 \mathrm{SW}_{\mathrm{H}}, \Delta t_{1 \mathrm{c}}=-\left(t_{1 \mathrm{~b}} /(\right.$ no. of complex points $\left.)\right)$ and $\Delta t_{1 \mathrm{~b}}=\Delta t_{1 \mathrm{a}}-\Delta t_{1 \mathrm{c}}$. All pulsed z-field gradients (PFGs) are sine-bell shaped with gradient recovery delay of $200 \mu \mathrm{s}$. The duration and strengths of the PFGs are: G1 $(1.0 \mathrm{~ms}, 15 \mathrm{G} / \mathrm{cm}) ; \mathrm{G} 2(1.0 \mathrm{~ms}, 40 \mathrm{G} / \mathrm{cm}) ; \mathrm{G} 3(1.0 \mathrm{~ms}, 4 \mathrm{G} / \mathrm{cm})$. The delays are: $\tau_{1}=2.2 \mathrm{~ms}, \tau_{2}=3.6 \mathrm{~ms}, \tau_{3}=4.4 \mathrm{~ms}, \tau_{4}=12.4 \mathrm{~ms}, \tau_{5}=6.2$ $\mathrm{ms}, \tau_{6}=0.6 \mathrm{~ms}, \tau_{7}=5.5 \mathrm{~ms}, \tau_{8}=2.3 \mathrm{~ms}$ and $\delta=1.2 \mathrm{~ms}$. Phase cycling: (i) for acquiring selectively the linear combination (Table 3 ): $\Omega\left({ }^{13} \mathrm{C}^{\alpha}\right.$ ) $+\Omega\left({ }^{1} \mathrm{H}^{\alpha}\right): \phi_{1}=x, y ; \phi_{2}=x, y ; \phi_{3}=x,-x ; \phi($ receiver $)=x,-x$; (ii) for acquiring selectively the linear combination $\Omega\left({ }^{13} \mathrm{C}^{\alpha}\right)-\Omega\left({ }^{1} \mathrm{H}^{\alpha}\right): \phi_{1}=x, y$; $\phi_{2}=x,-y ; \phi_{3}=x,-x ; \phi($ receiver $)=x,-x$.

$[1,14]$. Variations of these r.f. phases and the gradients can be used to appropriately select the cosine or sine modulation of ${ }^{15} \mathrm{~N}$ chemical shift for forming the linear combination. This is further illustrated below for $\mathrm{HN}$-detected out-andback triple resonance experiments employing the sensitivityenhanced scheme.

\section{NMR Data Collection}

The method is demonstrated with NMR spectra acquired for a $2.0 \mathrm{mM}$ sample of $\mathrm{u}-{ }^{13} \mathrm{C},{ }^{15} \mathrm{~N}$ labeled sample of $\mathrm{Z}$ domain $(7 \mathrm{kDa})[15,16]$. The following experiments were recorded: (i) $(3,2) \mathrm{D} \quad \mathrm{HACA}(\mathrm{CON}) \mathrm{HN}$, (ii) $(3,2) \mathrm{D}$ $\mathrm{HNNCO}(\mathrm{CA})$ and (iii) $(4,2) \mathrm{D} \mathrm{HNNCOCA}$. The r.f. pulse sequences are shown in Figs. (1-2) and have been derived from existing pulse sequences used for running $3 \mathrm{D}$ versions of these experiments without much modification. The r.f. pulse sequence used for $(3,2) \mathrm{D} \mathrm{HNNCO}(\mathrm{CA})$ is identical to $(4,2) \mathrm{D}$ HNNCOCA except that in the former case, the chemical shift evolution of CA $\left(t_{1}\right)$ is set to zero. All NMR experiments were performed at $25^{\circ} \mathrm{C}$ on a Bruker AVANCE $700 \mathrm{MHz}$ NMR spectrometer equipped with a cryogenic probe. The data were processed using NMRPIPE [17] and analyzed using XEASY [18]. Other than the standard 2D FT method, no other post-acquisition processing macros or scripts was used. All experiments were recorded with the minimum number of scans $(2$ scans for $(3,2) \mathrm{D}$ and 4 scans for $(4,2) \mathrm{D}$ GFT experiments).

\section{RESULTS AND DISCUSSION}

Figs. (3-5) shows the various GFT NMR spectra acquired for Z-domain using the r.f. pulse sequence shown in Figs. (1 and 2). The phase cycling steps used for these experiments are summarized in Table $\mathbf{3}$. The different linear combinations that get selected using the phases shown in Table $\mathbf{3}$ have been worked out in Table S1 of Supporting Information. In all the experiments, the existing 2 or more phase cycling steps in the r.f. sequence for water/artifact suppression have been utilized to incorporate the phase cycling procedure described above (e.g., the receiver phase cycling was set to $(X$, $-x$ ) in a 2 or 4 scans experiment). Since identical measurement times were used, the sensitivity was observed to be same across all the spectra. 


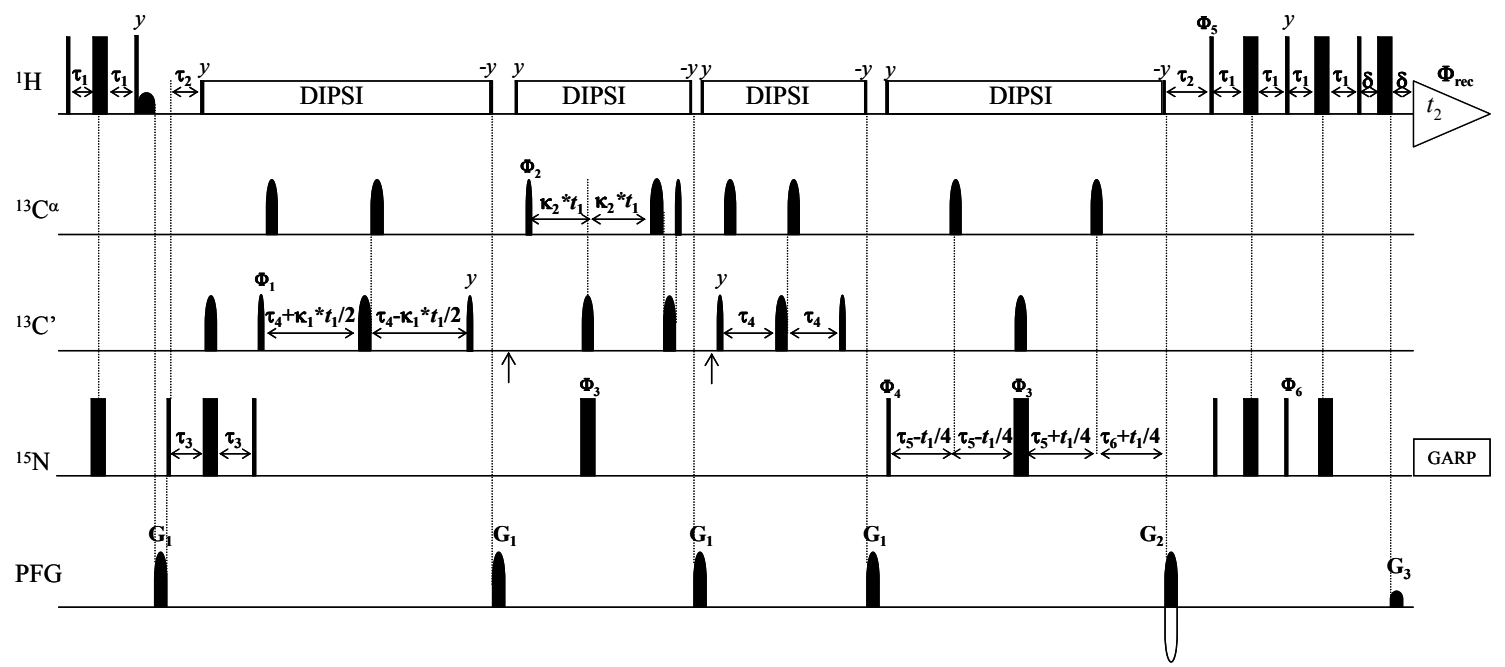

Fig. (2). R.f. pulse scheme of GFT $(4,2) \mathrm{D} H \mathrm{HNCOCA}$ and $(3,2) \mathrm{D} \mathrm{HNNCO}(\mathrm{CA})$ employing the gradient sensitivity enhanced mode of quadrature detection [14] for ${ }^{15} \mathrm{~N}$. Rectangular $90^{\circ}$ and $180^{\circ}$ pulses are indicated by thin and thick vertical bars, respectively, and phases are indicated above the pulses. Where no r.f. phase is marked, the pulse is applied along $x$. High-power $90^{\circ}$ pulse lengths are: $8.0 \mu$ s for ${ }^{1} \mathrm{H}, 14.5 \mu \mathrm{s}$ for ${ }^{13} \mathrm{C}$ and $37 \mu$ s for ${ }^{15} \mathrm{~N}$. The scaling factors: $\kappa_{1}=0.5$ and $\kappa_{2}=0.5$. For acquiring $(3,2) \mathrm{D} \mathrm{HNNCO}(\mathrm{CA}), \kappa_{2}=0$. The ${ }^{1} \mathrm{H}$ r.f. carrier is placed at the position of the solvent line at $4.7 \mathrm{ppm}$. The ${ }^{15} \mathrm{~N}$ carrier position is set to $119.5 \mathrm{ppm}$. The ${ }^{13} \mathrm{C}$ carrier is initially placed at $176 \mathrm{ppm}$ and switched to $54 \mathrm{ppm}$ before the first $90^{0}$ rf pulse on ${ }^{13} \mathrm{C}^{\alpha}$ (indicated by an arrow) and switched back to 54 ppm after the second $90^{0} \mathrm{rf}$ pulse on ${ }^{13} \mathrm{C}^{\alpha}$ (indicated by an arrow). All ${ }^{13} \mathrm{C}$ pulses are of Guassian cascade shape [1] having a pulse width of $320 \mu$ s for $90^{0}$ (on- or off- resonance) and $220 \mu$ s for $180^{\circ}$ (on- or off-resonance). DIPSI [1] (r.f = 3.2 kHz) is used for ${ }^{1} \mathrm{H}$ decoupling during ${ }^{13} \mathrm{C}-{ }^{13} \mathrm{C}$ and ${ }^{13} \mathrm{C}-{ }^{15} \mathrm{~N}$ polarization steps. GARP [1] is employed to decouple ${ }^{15} \mathrm{~N}$ (r.f. $=1.50 \mathrm{kHz}$ ) during acquisition. All pulsed z-field gradients (PFGs) are sine-bell shaped shaped with gradient recovery delay of $200 \mu \mathrm{s}$. The duration and strengths of the PFGs are: G1 (1.0 ms, $20 \mathrm{G} / \mathrm{cm}) ; \mathrm{G} 2(1.0 \mathrm{~ms}, 40 \mathrm{G} / \mathrm{cm})$; G3 (1.0 $\mathrm{ms}, 4 \mathrm{G} / \mathrm{cm})$. The delays are: $\tau_{1}=2.3 \mathrm{~ms}, \tau_{2}=5.5 \mathrm{~ms}, \tau_{3}=12.4 \mathrm{~ms}, \tau_{4}=4.4 \mathrm{~ms}, \tau_{5}=6.2 \mathrm{~ms}, \tau_{6}=0.7 \mathrm{~ms}, \delta=1.2 \mathrm{~ms}$. The sensitivity enhanced mode of data acquisition [1] is implemented by reversing the sign of the gradient G2 in concert with a $180^{\circ}$ phase shift of r.f. phase $\phi_{6}$. Phase cycling for acquiring selectively the linear combinations of chemical shifts (Table 3) in $(3,2) \mathrm{D} \mathrm{HNNCO}(\mathrm{CA})$ is: (i) $\Omega\left({ }^{15} \mathrm{~N}\right)+\kappa_{1} * \Omega\left({ }^{13} \mathrm{C}^{\prime}\right)$ : $\phi_{1}=$ $x, y ; \phi_{2}=x,-x ; \phi_{3}=x,-x ; \phi_{4}=x, y ; \phi_{5}=x ; \phi_{6}=-y ; \phi($ receiver $)=x,-x ;($ ii $) \Omega\left({ }^{15} \mathrm{~N}\right)-\kappa_{1} * \Omega\left({ }^{13} \mathrm{C}^{\prime}\right): \phi_{1}=x, y ; \phi_{2}=x,-x ; \phi_{3}=x,-x ; \phi_{4}=x,-y ; \phi_{5}=x$; $\phi_{6}=-y ; \phi($ receiver $)=x,-x$. Phase cycling for selectively acquiring the different linear combinations of chemical shifts $($ Table 3$)$ in $(4,2) \mathrm{D}$ HNNCOCA is: (i) $\Omega\left({ }^{15} \mathrm{~N}\right)+\Omega\left({ }^{13} \mathrm{C}^{\prime}\right)+\Omega\left({ }^{13} \mathrm{C}^{\alpha}\right): \phi_{1}=x, y,-x,-y ; \phi_{2}=x, y, y,-x ; \phi_{3}=x,-x ; \phi_{4}=x, x, y, y ; \phi_{5}=x, x,-x,-x ; ; \phi_{6}=-y,-y, y, y$; $\phi$ (receiver) $=x,-x$; (ii) $\Omega\left({ }^{15} \mathrm{~N}\right)+\Omega\left({ }^{13} \mathrm{C}^{\prime}\right)-\Omega\left({ }^{13} \mathrm{C}^{\alpha}\right): \phi_{1}=x, y,-x,-y ; \phi_{2}=-x, y, y, x ; \phi_{3}=x,-x ; \phi_{4}=x, x, y, y ; \phi_{5}=x, x,-x,-x ; ; \phi_{6}=-y,-y, y, y$; $\phi($ receiver $)=x,-x$; (iii) $\Omega\left({ }^{15} \mathrm{~N}\right)-\Omega\left({ }^{13} \mathrm{C}^{\prime}\right)+\Omega\left({ }^{13} \mathrm{C}^{\alpha}\right): \phi_{1}=x, y,-x,-y ; \phi_{2}=-x, y, y, x ; \phi_{3}=x,-x ; \phi_{4}=x, x,-y,-y ; \phi_{5}=x, x,-x,-x ; ; \phi_{6}=-y,-y, y, y$; $\phi($ receiver $)=x,-x$; (iv) $\Omega\left({ }^{15} \mathrm{~N}\right)+\Omega\left({ }^{13} \mathrm{C}^{\prime}\right)-\Omega\left({ }^{13} \mathrm{C}^{\alpha}\right): \phi_{1}=x, y,-x,-y ; \phi_{2}=x, y, y,-x ; \phi_{3}=x,-x ; \phi_{4}=x, x,-y,-y ; \phi_{5}=x, x,-x,-x ; \phi_{6}=-y,-y, y, y$; $\phi($ receiver $)=x,-x$.
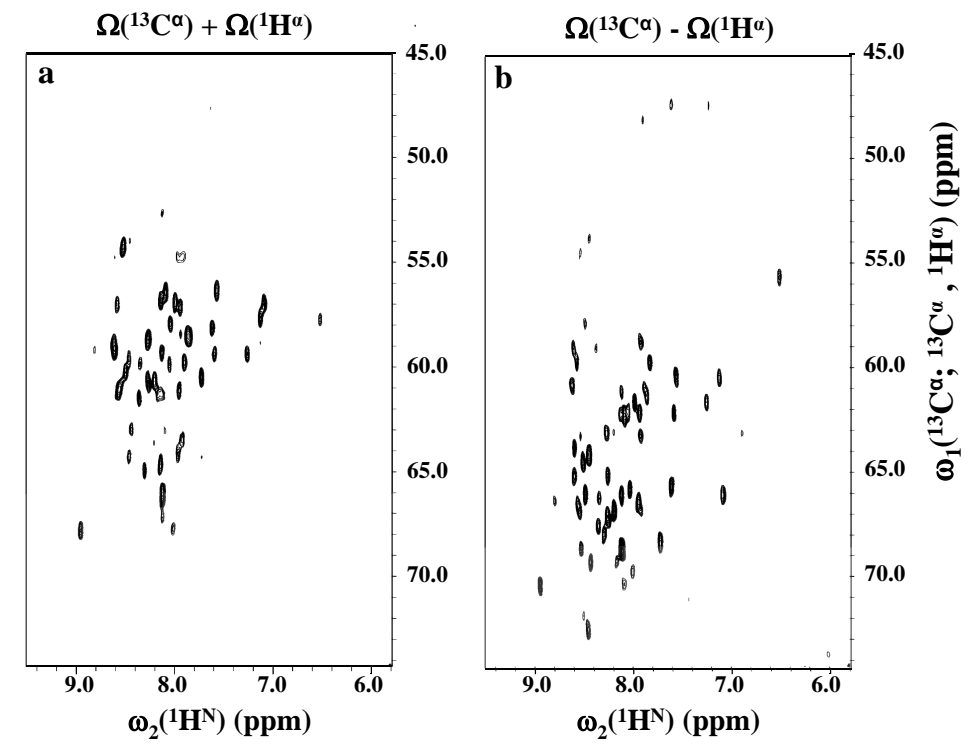

Fig. (3). The (3,2)D HACA(CON)HN GFT spectra acquired for Z-domain using the r.f pulse sequence of Fig. (1) and using the specific phase cycling procedure outlined in Table $\mathbf{3}$. The linear combination of chemical shifts observed in sub-spectra (a) and (b) are indicated on the top. These spectra (a) and (b) were acquired separately with 2 scans/transients each resulting in total measurement time of $\sim 10$ minutes. Other than a routine 2D Fourier transformation step, no other post-acquisition data processing procedure was used. 

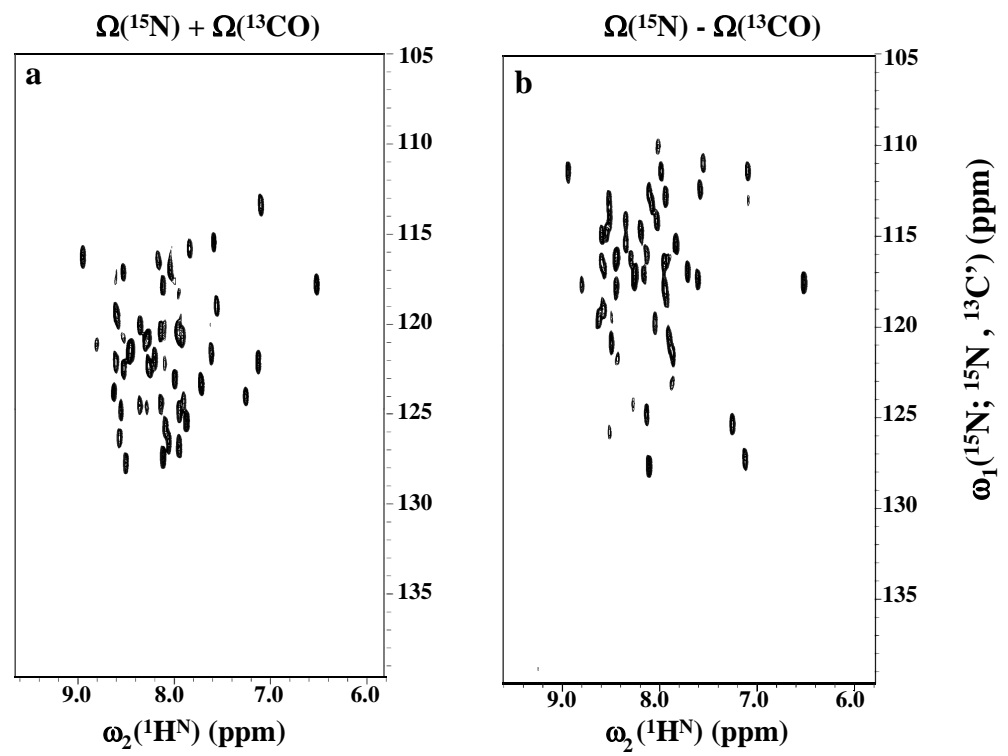

Fig. (4). The (3,2)D HNNCO $(\mathrm{CA})$ GFT spectra acquired for Z-domain using the r.f pulse sequence of Fig. (2) and using the specific phase cycling procedure outlined in Table $\mathbf{3}$. The linear combination of chemical shifts observed in sub-spectra (a) and (b) are indicated on the top. These spectra (a) and (b) were acquired separately with 2 scans/transients each resulting in total measurement time of $\sim 10$ minutes. Other than a routine $2 \mathrm{D}$ Fourier transformation step, no other post-acquisition data processing procedure was used.
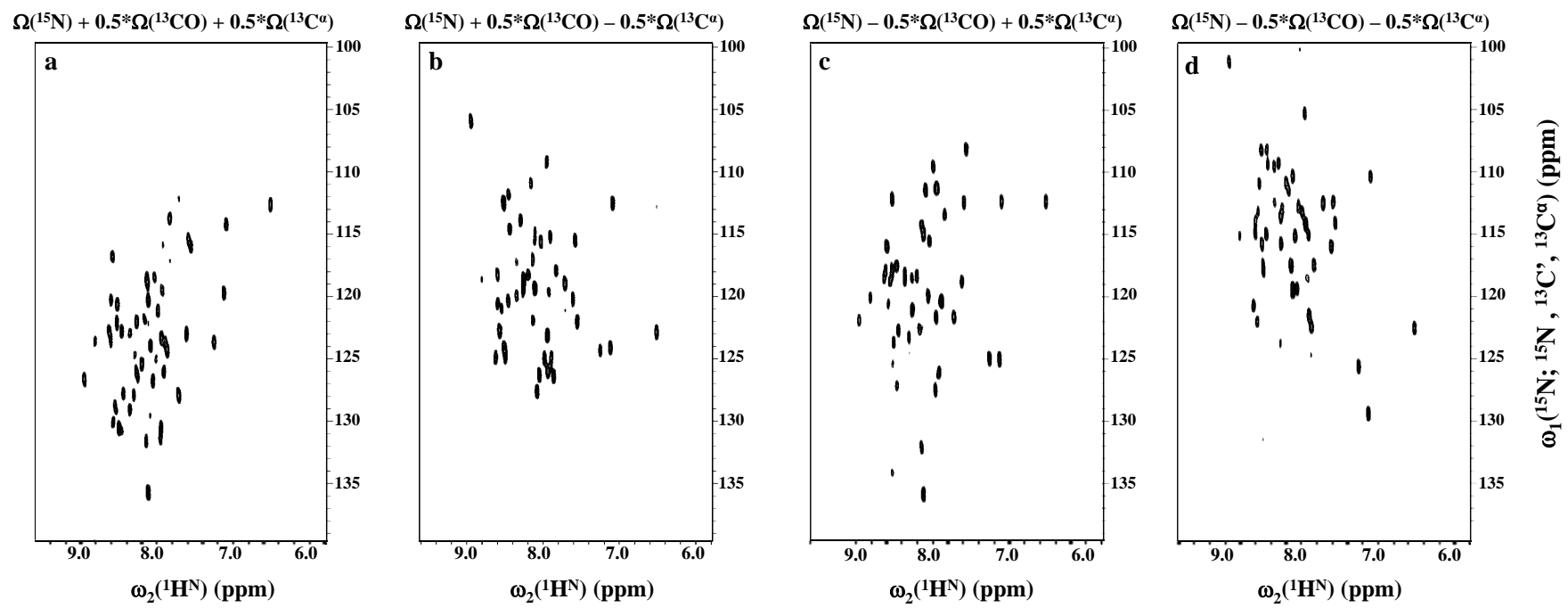

Fig. (5). The (4,2)D HNNCOCA GFT spectra acquired for Z-domain using the r.f pulse sequence of Fig. (2) and using the specific phase cycling procedure outlined in Table $\mathbf{3}$. The linear combination of chemical shifts observed in sub-spectra (a)-(d) is indicated on the top. These spectra were acquired separately with 4 scans/transients each resulting in total measurement time of $\sim 40$ minutes. Other than a routine 2D Fourier transformation step, no other post-acquisition data processing procedure was used.

\section{Applications Where Only a Few Linear Combinations are Desired}

In many instances, acquiring only one or a few linear combinations out of a set of $2^{K}$ shift multiplets may be sufficient. Alternatively, one may desire a specific set of linear combination to be observed (example: In a $(3,2) \mathrm{D}$ projection experiment: $\kappa_{\mathrm{A}} * \Omega_{\mathrm{A}}+\kappa_{\mathrm{B}} * \Omega_{\mathrm{B}}$ and $\kappa_{\mathrm{A}} * \Omega_{\mathrm{A}}+\kappa_{\mathrm{B}}{ }^{\prime} * \Omega_{\mathrm{B}}$ where $\kappa_{A} \neq \kappa_{A}{ }^{\prime} ; \kappa_{B} \neq \kappa_{B}{ }^{\prime}$ may be desired and not necessarily $\left.\kappa_{\mathrm{A}} * \Omega_{\mathrm{A}} \pm \kappa_{\mathrm{B}} * \Omega_{\mathrm{B}}\right)$. Thus, it is desirable to remove the restriction of acquiring an entire data set comprising $2^{K}$ shift combinations and allow any desired linear combination of shifts to be selectively chosen for detection. While the methodology described here is applicable to all projection NMR experiments in general, a few examples are given here to illustrate this point.

1. In the (5,3)D $G^{2} F T$ NMR experiments [19] used for resonance assignments of systems with high chemical shift degeneracy such as membrane proteins [11], two sets of spins are jointly sampled in two different dimensions of a ND NMR experiment. One of the GFT dimensions serves solely to provide increased resolution which is achieved by joint sampling of two chemical shifts (e.g., ${ }^{15} \mathrm{~N}$ and $\left.{ }^{13} \mathrm{C}^{\prime}\right)$. Thus, only a par- 
Table 3. Phase Cycling Used for Selective Observation of Different Linear Combination of Chemical Shifts

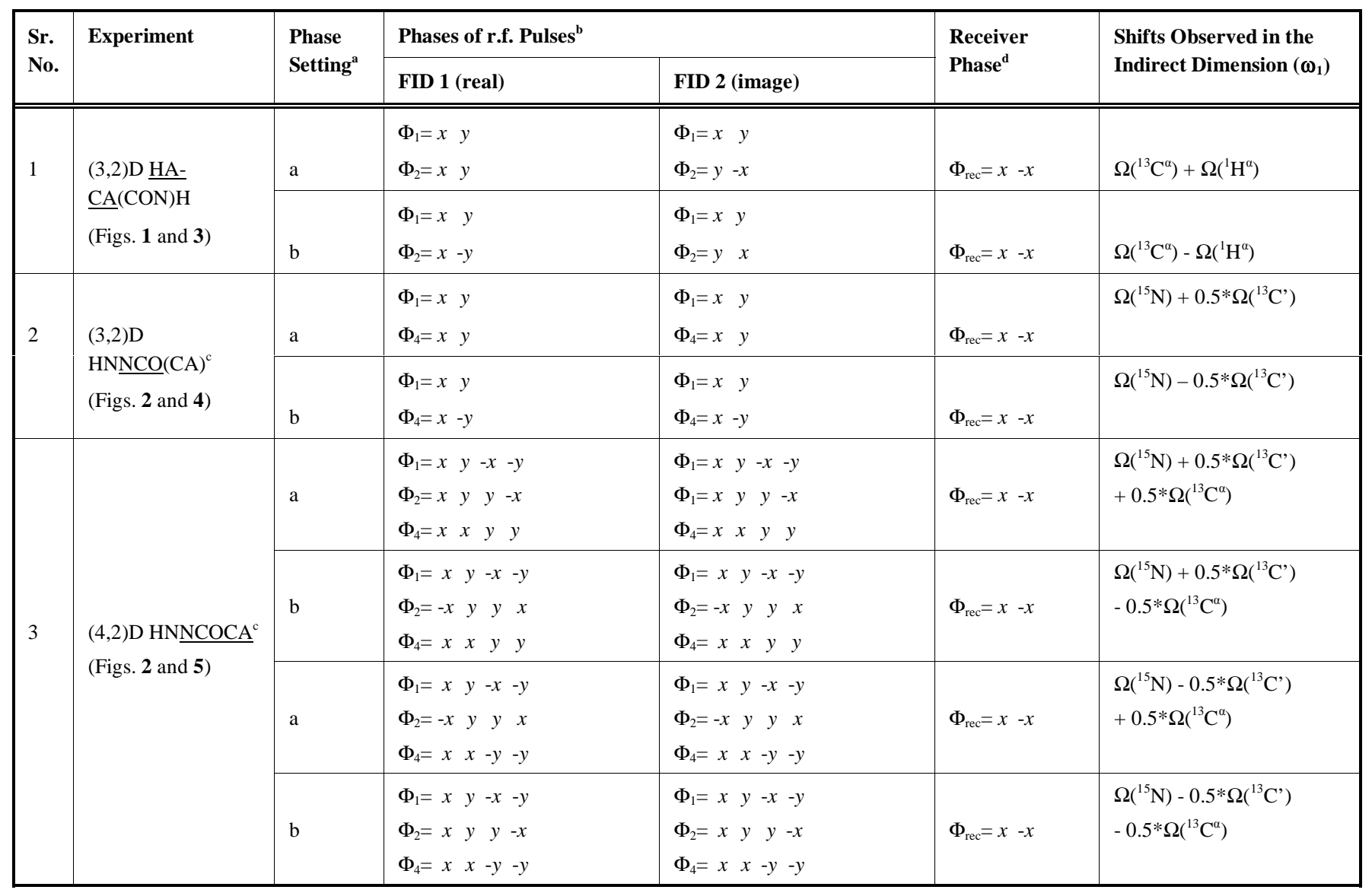

a'The labels ('a','b', 'c' and 'd') correspond to spectra shown in Figs. (3-5).

${ }^{\mathrm{b}}$ The r.f. phases $\left(\Phi_{i}\right)$ correspond to those shown in their respective r.f. pulse sequences (Figs. 1-2).

${ }^{c}$ In the case of r.f. pulse sequences employing sensitivity enhanced mode of data acquisition, the FID for the imaginary part is acquired with phase cycling of the appropriate r.f. phases and gradients $[1,14]$. The phase cycling of r.f. phase $\Phi_{4}$ is kept constant.

For example, in Fig. (2), the phase, $\Phi_{6}$, is changed by $180^{\circ}$ simultaneously with inversion of gradient G2. See also Table S1.

${ }^{\mathrm{d}}$ A two step receiver phase cycle $\left(0^{0}, 180^{\circ}\right)$ was used for water suppression allowing data to be recorded with 2 or 4 scans/transients.

ticular linear combination (either $\Omega\left({ }^{15} \mathrm{~N}\right)+\kappa \Omega\left({ }^{13} \mathrm{C}^{\prime}\right)$ or $\left.\Omega\left({ }^{15} \mathrm{~N}\right)-\kappa \Omega\left({ }^{13} \mathrm{C}^{\prime}\right)\right)$ which provides more dispersion in chemical shift can be chosen in the resolving dimension and the need to acquire both the sums and differences of chemical shifts may not be necessary. Alternatively, two different combination of the type $\Omega\left({ }^{15} \mathrm{~N}\right)+\kappa \Omega\left({ }^{13} \mathrm{C}^{\prime}\right)$ and $\left.\Omega\left({ }^{15} \mathrm{~N}\right)+\kappa^{\prime} \Omega\left({ }^{13} \mathrm{C}^{\prime}\right)\right)\left(\kappa \neq \kappa^{\prime}\right)$ may afford better resolution than $\Omega\left({ }^{15} \mathrm{~N}\right) \pm \kappa \Omega\left({ }^{13} \mathrm{C}^{\prime}\right)$ and hence may be desired. The combination which has better dispersion can be decided by recording quick 2D planes with different linear combinations and the appropriate one can be used for recording the longer $(5,3) \mathrm{D}$ experiment.

2. A second example is the application of GFT NMR for rapid measurement of ${ }^{3} \mathrm{~J}_{\mathrm{HNHNa}}$ in proteins [20]. The coupling constants are measured by recording a $(3,2) \mathrm{D}$ HNHA experiment. If this experiment is recorded after completion of the backbone resonance assignments, then the spectra comprising peaks at one of the linear combination of shifts (either $\Omega\left({ }^{15} \mathrm{~N}\right.$ ) $+\kappa\left({ }^{1} \mathrm{H}^{\alpha} /{ }^{1} \mathrm{H}^{\mathrm{N}}\right)$ or $\left.\Omega\left({ }^{15} \mathrm{~N}\right)-\kappa\left({ }^{1} \mathrm{H}^{\alpha} /{ }^{1} \mathrm{H}^{\mathrm{N}}\right)\right)$ in the indirect dimension is sufficient to measure the coupling constants resulting in savings in measurement time.
3. Another example is that of reconstruction of $N \mathrm{D}$ NMR spectra from a set of projections [7]. It has been noted that depending upon spectral complexity, it is possible to reconstruct a spectrum with less than the $2^{k}$ GFT planes needed to determine chemical shifts [7, 21]. Thus, for a 3D spectrum of low complexity, the two orthogonal projections at $0^{\circ}$ and $90^{\circ}$ and an additional projection at $45^{0}$ may be sufficient. This implies that in certain cases only 3 spectra comprising chemical shifts at $\Omega_{\mathrm{A}}, \Omega_{\mathrm{B}}$ and $\Omega_{\mathrm{A}}+\Omega_{\mathrm{B}}$ may suffice and an additional combination, $\Omega_{\mathrm{A}}-\Omega_{\mathrm{B}}$ (projection at angle $-45^{0}$ ) need not be acquired. This is further beneficial for reconstructions of higher dimensional spectra. For instance, if projections at angles $\left(\alpha_{1},-\beta_{1}, \pm \gamma\right)$ and $\left(\alpha_{2},-\beta_{2}, \pm \gamma\right)$ is needed for a analyzing a $5 \mathrm{D}$ experiment, an entire data set comprising peaks at $\left( \pm \alpha_{1}\right.$, $\left.\pm \beta_{1}, \pm \gamma\right)$ and $\left( \pm \alpha_{2}, \pm \beta_{2}, \pm \gamma\right)$ need not be acquired and the measurement time can be saved by acquiring only the desired projections. The measurement time thus released can be efficiently utilized towards acquiring a larger number of projections at specific angles rather than sampling at undesired angles. This will benefit automated methods such as HIFI-NMR [8] 
and APSY [9], wherein a large number of projections at any desired angle(s) can be selectively detected.

\section{Potential for Reducing Measurement Time}

By selecting only the desired linear combination of chemical shifts, there is a potential for reducing the total measurement time required for collecting the data. This is explained as follows. The minimum number of steps in the phase cycling required for method described here will depend on the number of spins that are jointly sampled. In the case of $K+1$ jointly sampled spins, a minimum number of $2^{K+1}$ phase cycling steps are required for each increment in the indirect dimension (e.g., four scans for 2 jointly sampled spins; see Tables 1-3). This includes 2 scans for quadrature detection of the centre shift. In comparison, the current implementations of all projection NMR experiments require for each increment: (i) $2^{K+1}$ steps for phase cycling for observing all the $2^{K}$ shift combinations ([5-7]; see Eq. 1) and (ii) additional $2^{M}(M \geq 1)$ scans or transients if water/artifact suppression and/or increased sensitivity is needed resulting in a total number of $2^{K+1+M}$ scans. Thus, if only $L$ out of $2^{K}$ linear combinations are desired, data acquisition using the phase cycling method described here is faster by a factor of $2^{M} / L$. The minimum gain is a factor of $2(M=1 ; L=1)$ since a minimum of 2-step phase cycling is almost always used for good water suppression [1]. If more than 2 scans are used for water/artifact suppression, the gain in measurement time by detection of select combination of chemical shifts is even higher.

For example, in a $(3,2) \mathrm{D}$ experiment, 4 FIDs with all possible cosine/sine modulation of the two jointly sampled chemical shifts are acquired with each FID comprising a minimum of 2 scans/transients per complex point (for efficient water line suppression [1]) resulting in a total 8 scans/transients to get the two linear combinations: $\kappa_{\mathrm{A}} * \Omega_{\mathrm{A}} \pm \kappa_{\mathrm{B}} * \Omega_{\mathrm{B}} \quad$ (Eq 1). If only one combination $\kappa_{\mathrm{A}} * \Omega_{\mathrm{A}}+\kappa_{\mathrm{B}} * \Omega_{\mathrm{B}}$ is desired, 2 FIDs with 2 scans each can be used with the method described herein (Table 1) to get the desired combination in a total of 4 scans. While the measurement time for acquiring both linear combinations $\left(\kappa_{\mathrm{A}} * \Omega_{\mathrm{A}} \pm \kappa_{\mathrm{B}} * \Omega_{\mathrm{B}}\right)$ is same in the two methods (i.e., the existing methods [2-5] and the phase cycling approach described here), selective detection of desired linear combination of shifts as described here releases measurement time to acquire, if required, a different combination of chemical shift, say, $\kappa_{A}{ }^{\prime} * \Omega_{A}+\kappa_{B}{ }^{\prime} * \Omega_{B}\left(\kappa_{A} \neq \kappa_{A}{ }^{\prime} ; \kappa_{B} \neq \kappa_{B}{ }^{\prime}\right)$. In other words, one can acquire two spectra with combinations: $\kappa_{\mathrm{A}} * \Omega_{\mathrm{A}}+\kappa_{\mathrm{B}} * \Omega_{\mathrm{B}}$ and $\kappa_{\mathrm{A}}{ }^{\prime} * \Omega_{\mathrm{A}}+\kappa_{\mathrm{B}}, * \Omega_{\mathrm{B}}$ in the same measurement as acquiring $\kappa_{\mathrm{A}} * \Omega_{\mathrm{A}} \pm \kappa_{\mathrm{B}} * \Omega_{\mathrm{B}}$. As explained in the previous section, carefully chosen $\kappa_{\mathrm{A}} * \Omega_{\mathrm{A}}+\kappa_{\mathrm{B}} * \Omega_{\mathrm{B}}$ and $\kappa_{\mathrm{A}}{ }^{\prime} * \Omega_{\mathrm{A}}+\kappa_{\mathrm{B}}{ }^{\prime} * \Omega_{\mathrm{B}}$ may offer more information/resolution in certain cases than $\kappa_{\mathrm{A}} * \Omega_{\mathrm{A}} \pm \kappa_{\mathrm{B}} * \Omega_{\mathrm{B}}$ and hence measurement time can be saved by avoiding acquisition of 4 combinations: $\kappa_{\mathrm{A}} * \Omega_{\mathrm{A}} \pm \kappa_{\mathrm{B}} * \Omega_{\mathrm{B}}$ and $\kappa_{\mathrm{A}}{ }^{\prime} * \Omega_{\mathrm{A}} \pm \kappa_{\mathrm{B}}{ }^{\prime} * \Omega_{\mathrm{B}}$ as required in the current implementations. Note that the experiment is carried out within the 2 scans required for efficient water line suppression and no additional phase cycling steps are required. Further, the sensitivity of the experiments with respect to the existing implementations is not compromised and will only depend on the total measurement time used. While we propose this method for selecting one or a few linear combination of chemical shifts, it can also be used for observing all the $2^{K}$ combinations as done in current implementations.

\section{CONCLUSIONS}

We have described a method of phase cycling to select any desired linear combination of chemical shifts or projections for detection out of all possible shift combinations. This method avoids the need to use post-acquisition data processing and removes the restriction of acquiring all possible $2^{K}$ shift combinations, thereby benefiting experiments wherein a few of the particular linear combinations are desired. Further reductions in measurement time can be achieved by combining this approach with other methods for fast data collection such as the recently proposed simultaneous phase cycling approach based on slice selective excitation [22] and/or longitudinal ${ }^{1} \mathrm{H}$ relaxation optimization $[6$, 23]. In the case of large molecular weight proteins, these experiments can be combined with deuteration and/or TROSY [24]. Taken together, this approach will foster new applications in projection NMR spectroscopy for rapid resonance assignments and structure determination.

\section{ACKNOWLEDGEMENTS}

The NMR facility provided at NMR Research Centre at IISc, Bangalore supported by the Department of Science and Technology (DST) is gratefully acknowledged. HSA acknowledges research support from DAE-BRNS, DST-Fast track and DST-SERC projects. We thank Drs. Montelione G and Aramini J, Rutgers University, for providing the Zdomain protein sample. MS acknowledges fellowship from CSIR, India.

\section{SUPPLEMENTARY MATERIAL}

Supplementary material can be viewed at www.bentham.org/open/tomrj

\section{REFERENCES}

[1] Cavanagh C, Fairbrother WJ, Palmer AG, Skelton NJ. Protein NMR Spectroscopy. Academic Press: San Diego 2007.

[2] Atreya HS, Szyperski T. Rapid NMR data collection. Methods Enzymol 2005; 394: 78-108.

[3] Szyperski T, Atreya HS. Principles and applications of GFT projection NMR spectroscopy. Magn Reson Chem 2006; 44: S51-S60.

[4] Szyperski T, Yeh DC, Sukumaran DK, Moseley HNB, Montelione GT. Reduced-dimensionality NMR spectroscopy for highthroughput protein resonance assignment. Proc Natl Acad Sci USA 2002; 99: 8009-14.

[5] Kim S, Szyperski T. GFT NMR, a new approach to rapidly obtain precise high-dimensional spectral information. J Am Chem Soc 2003; 125: 1385-93.

[6] Atreya HS, Szyperski T. G-matrix Fourier transform NMR spectroscopy for complete protein resonance assignment. Proc Natl Acad Sci USA 2004; 101: 9642-7.

[7] Kupče E, Freeman R. Projection-reconstruction technique to speed up multidimensional NMR spectroscopy. J Am Chem Soc 2004; 126: 6429-40.

[8] Eghbalnia HR, Bahrami A, Tonelli M, Hallenga K, Markley JL. High-resolution iterative frequency identification for NMR as a general strategy for multidimensional data collection. J Am Chem Soc 2005; 127: 12528-36.

[9] Hiller S, Fiorito F, Wüthrich K, Wider G. Automated projection NMR spectroscopy (APSY). Proc Natl Acad Sci USA 2005; 102 : 10876-81.

[10] Liu GH, Shen Y, Atreya HS, et al. NMR data collection and analysis protocol for high-throughput protein structure determination. Proc Natl Acad Sci USA 2005; 102: 10487-92. 
[11] Zhang Q, Atreya HS, Kamen DE, Girvin ME, Szyperski T. GFT Projection NMR based resonance assignment of membrane proteins: application to subunit $\mathrm{c}$ of $E$. coli $\mathrm{F}_{1} \mathrm{~F}_{0}$ ATP synthase in LPPG micelles. J Biomol NMR 2008; 40: 157-63.

[12] Hiller S, Wasmer C, Wider G, Wüthrich K. Sequence-specific resonance assignment of soluble nonglobular proteins by $7 \mathrm{D}$ APSY-NMR spectroscopy. J Am Chem Soc 2007; 129: 10823-8.

[13] States DJ, Haberkorn RT, Ruben DJ. A two-dimensional nuclear overhauser experiment with pure absorption phase in four quadrants. J Magn Reson 1982; 48: 286-92.

[14] Kay LE, Keifer P, Saarinen T. Pure absorption gradient enhanced heteronuclear single quantum coherence spectroscopy with increased sensitivity. J Am Chem Soc 1992; 114: 10663-5.

[15] Tashiro M, Tejero R, Zimmerman DE, Bernardo C, Nilsson B, Montelione GT. High-resolution solution NMR structure of the Z domain of staphylococcal protein A. J Mol Biol 1997; 273: 573-90.

[16] Zheng D, Aramini JM, Montelione, GT. Validation of helical tilt angles in the solution NMR structure of the Z domain of Staphylococcal protein A by combined analysis of residual dipolar coupling and NOE data. Protein Sci 2004; 13: 549-54.

[17] Delaglio F, Grzesiek S, Vuister GW, Zhu G, Pfeifer J, Bax A. Nmrpipe - a multidimensional spectral processing system based on unix pipes. J Biomol NMR 1995; 6: 277-93.
[18] Bartels C, Xia TH, Billeter M, Güntert P, Wüthrich K. The program XEASY for computer-supported NMR spectral analysis of biological macromolecules. J Biomol NMR 1995; 6: 1-10.

[19] Atreya HS, Eletsky A, Szyperski T. Resonance assignment of proteins with high shift degeneracy based on 5D spectral information encoded in $\mathrm{G}^{2} \mathrm{FT}$ NMR experiment. J Am Chem Soc 2005; 127: 4554-5.

[20] Barnwal RP, Rout AK, Chary KVR, Atreya HS. Rapid measurement of ${ }^{3} \mathrm{~J}_{\mathrm{HNH} \alpha}$ and ${ }^{3} \mathrm{~J}_{\mathrm{NH} \beta}$ in proteins. J Biomol NMR 2007; 39: 259. 63.

[21] Venters RA, Coggins BE, Kojetin D, Cavanagh J, Zhou P. (4,2)D Projection-reconstruction experiments for protein backbone assignment: application to human carbonic anhydrase II and calbindin D28K. J Am Chem Soc 2005; 127: 8785-95.

[22] Parish DM, Szyperski T. Simultaneously cycled NMR spectroscopy. J Am Chem Soc 2008; 130: 4925-33.

[23] Pervushin K, Vogeli B, Eletsky A. Longitudinal ${ }^{1} \mathrm{H}$ relaxation optimization in TROSY NMR spectroscopy. J Am Chem Soc 2002; 124: 12898-902.

[24] Pervushin K, Riek R, Wider G, Wüthrich K. Attenuated T-2 relaxation by mutual cancellation of dipole-dipole coupling and chemical shift anisotropy indicates an avenue to NMR structures of very large biological macromolecules in solution. Proc Natl Acad Sci USA 1997; 94: 12366-71.

(C) Swain and Atreya; Licensee Bentham Open .

This is an open access article licensed under the terms of the Creative Commons Attribution Non-Commercial License (http://creativecommons.org/licenses/by-nc/3.0/) which permits unrestricted, non-commercial use, distribution and reproduction in any medium, provided the work is properly cited. 